\title{
Éduquer à l'échelle du monde
}

Entretien avec Mamadou Diouf (Propos recueillis par François Durpaire et Béatrice Mabilon-Bonfils, 2013)

\section{Mamadou Diouf}

\section{(2) OpenEdition}

1 Journals

Édition électronique

URL : http://journals.openedition.org/trema/3033

DOI : $10.4000 /$ trema.3033

ISSN : 2107-0997

Éditeur

Faculté d'Éducation de l'université de Montpellier

\section{Édition imprimée}

Date de publication : 1 décembre 2013

Pagination : 4 - 15

ISSN : 1167-315X

\section{Référence électronique}

Mamadou Diouf, «Éduquer à l'échelle du monde », Tréma [En ligne], 40 | 2013, mis en ligne le 01 décembre 2015, consulté le 07 mai 2019. URL : http://journals.openedition.org/trema/3033 ; DOI : 10.4000/trema.3033

Ce document a été généré automatiquement le 7 mai 2019.

Trema 


\section{Éduquer à l'échelle du monde}

Entretien avec Mamadou Diouf (Propos recueillis par François Durpaire et Béatrice Mabilon-Bonfils, 2013)

\section{Mamadou Diouf}

En quoi peut-on dire que nous sommes dans un temps du monde? Est-ce que nous pouvons dire aujourd'hui que nous sommes dans le temps du monde? 
1 M. D.: Oui. Je pense qu'on peut dire qu'aujourd'hui, on est dans le temps du monde pour plusieurs raisons. La première raison est purement technologique. Les sciences de l'information et de la communication ont changé la géographie du monde et ont créé la connexion - c'est le mot le plus important de notre vocabulaire. On se connecte. Il y a possibilité de se connecter. Il y a toute une série d'infrastructures et de bibliothèques qui ont créé un espace à la fois d'accumulation et de circulation.

La deuxième raison, plus importante, y est associée. La révolution technologique est

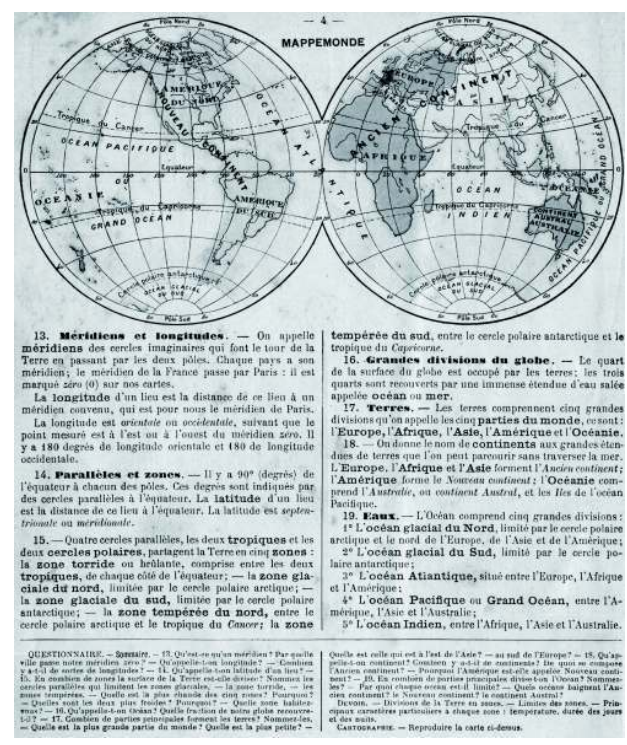
probablement la fin de cela. Nous vivons dans une temporalité qui a réduit à leur plus simple expression les temporalités qui ont existé. Avant le XVe siècle, avant la période des grandes découvertes, il y avait des temporalités du temps du monde qui étaient portées par des nations différentes, africaines, indiennes ou européennes. L'Europe n'était qu'une nation parmi d'autres. Sa temporalité était indigène. Ces temporalités indigènes ont été réduites en miettes.

D'une part, au XVe siècle, avec les grandes découvertes, l'Europe va à l'assaut du monde. D'autre part, et c'est très important d'un point de vue intellectuel, il se trouve que les Lumières ont opéré des réorganisations et des réaménagements importants. Il n'y a plus qu'un seul temps du monde. Ce temps du monde européen efface l'Europe comme lieu particulier. Cela devient l'espace du monde. Et l'Européen devient l'Homme, et son histoire devient l'Histoire - avec un grand H. Ce temps du monde a été le temps des grandes découvertes, le temps des empires coloniaux, le temps de l'exploitation coloniale et le temps de la réduction de toutes les civilisations à une seule. Quand on parle alors de civilisation, ce n'est pas au pluriel. On parle d'une civilisation unique, la civilisation de l'homme, c'est-à-dire la civilisation européenne.

4 Et c'est ce temps du monde qui est en train d'exploser avec les nouvelles technologies et qui a explosé dès la fin de la Seconde Guerre mondiale quand les sociétés colonisées ont commencé à produire leur propre récit. Le récit de l'universel, le récit européen, est contesté maintenant dans les périphéries.

5 Parmi les trois grandes révolutions du XVIIIe siècle, la plus importante est la révolution haïtienne. Cette révolution se fixe comme objectif de réaliser le récit des Lumières. Ce n'est plus la révolution américaine. Ce n'est pas non plus la Révolution française. Et, pourtant, la réalisation du défi de la révolution haïtienne se réalise probablement dans les années quatre-vingts quand, pour la première fois, des sociétés décolonisées après la Seconde Guerre mondiale ont commencé à produire leur propre récit, à contester l'universel européen et ont produit un temps du monde désormais pluriel. C'est ce qui est intéressant. C'est un temps du monde qui se compose de plusieurs temporalités qui se chevauchent, se juxtaposent mais entrent aussi en compétition. Et, comme toutes les autres cultures, l'Europe devient une culture indigène. 
Nous voudrions aborder une question un peu plus personnelle sur les mutations que vous avez perçues entre l'école que vous avez connue et celle d'aujourd'hui. Quelle est votre perception des évolutions de l'école au Sénégal jusqu'à l'école d'aujourd'hui ?

6 M. D. : Les mutations sont importantes. J'ai été formé comme un petit Français - ce que j'étais. C'est une chose qu'il est important de comprendre. Je suis né dans une ville française, où les gens étaient citoyens depuis 1880. Ils avaient les mêmes droits et les mêmes responsabilités que les Français. Ils élisaient un député à l'Assemblée nationale. Les habitants des quatre communes (Saint-Louis, Rufisque, Dakar et Gorée) - je suis de Rufisque - ont élu leur premier député noir en 1914. Cet homme a joué un rôle très important. Il a joué sur deux approches. Une première approche nous ramène à notre première question. La politique d'assimilation n'est pas une politique coloniale. Elle est une revendication, en particulier, des gens des quatre communes qui veulent être éduqués à leur propre gré. "Ils veulent assimiler sans être assimilés » - pour reprendre les mots de Senghor. Cela veut dire que ces populations ont une claire conscience de ce qu'ils sont et de ce qui les différencie des Français. C'est un mouvement intellectuel. Ces gens veulent savoir.

7 Dans l'un des plus grands romans sénégalais, L'Aventure ambiguë, publié en 1962, de Cheikh Hamidou Kane, il y a un débat extraordinaire entre le chef de la communauté des Diallobé et sa sœur, la Grande royale. La discussion tourne autour de l'éducation. Faut-il choisir une éducation coranique ou occidentale? Le maître coranique refuse une éducation occidentale. En particulier, il voudrait former le fils du chef pour devenir le maître. La Grande royale y est opposée. Pourquoi ? Elle dit qu'il faut envoyer les enfants à l'école des Blancs pour apprendre à gagner sans avoir raison. La technologie n'efface pas toute la question du maintien de connaissances culturelles et d'attributs qui sont propres aux sociétés.

8 J'ai été éduqué dans cette tension, comme mon père l'a été. Mon père est allé hors de sa société, hors de sa communauté pour apprendre. De ce fait, cet homme n'a jamais parlé la langue de ses parents. Il est allé ensuite à l'école française. Je n'ai pas eu cette éducation. J'ai reçu une éducation qui était parallèle, mais dans laquelle l'école coranique était secondaire. C'était une école coranique qui n'était pas comme celle de mon père où on sortait de son milieu pour aller vivre ailleurs et où on mendiait. C'était quelque chose de très dur. Pour lui, aller à l'école française a constitué un double déplacement: un déplacement purement communautaire (il n'était plus dans le milieu de ses parents) et un déplacement intellectuel. Il a appris le Coran et à lire l'arabe. Il est passé à une autre langue.

9 Moi, je suis allé à l'école française. Et aller à l'école française voulait dire que, le soir, on allait à l'école coranique. Mais il s'agissait d'une école coranique urbaine. Mon père allait à une école coranique rurale. Dans ces écoles, qui étaient assez fantastiques, non seulement nous avons appris comme les petits Français, mais le rapport que nous avions avec la langue a changé le type d'éducation. Mon père parlait français. Ma mère ne parlait pas français. En fait, la chose que détestait le plus ma mère, c'était que les gens parlent français. Parler français voulait dire que l'on disait des choses mauvaises sur elle. Quand j'arrivais à la maison, le français n'était plus la langue que j'utilisais. Mais quand j'allais à l'école, on m'interdisait de parler la langue que je parlais avec ma mère.

10 En fait, le processus d'adaptation à cette école a été très difficile pour beaucoup de gens. Pour moi, je ne peux pas expliquer pourquoi, mais j'aimais aller à l'école (rires). Je ne peux pas expliquer pourquoi j'ai été capable de bien faire. C'est vrai que mon père m'a 
aidé parce qu'il parlait français. J'avais aussi un frère qui allait à l'école. Mes sœurs allaient à l'école aussi. Mais la tension a toujours été résolue par une chose. C'est probablement l'une des caractéristiques des habitants des quatre communes: ils ont choisi eux-mêmes la manière d'assimiler. Je participais de cette culture en tant que citoyen d'Empire. Quelque part, j'avais des capacités citoyennes que les autres Sénégalais n'avaient pas. En fait, les gens des quatre communes regardaient les autres comme les Français nous regardaient: c'était des primitifs. De ce fait, la compétition avec les Français est devenue la chose la plus importante. Il s'agissait de leur montrer que nous avions un script, une écriture, l'arabe, et que depuis des siècles les gens de ces villes ou de ces régions étaient capables de philosopher. Ils lisaient Aristote et Platon en arabe. Ils n'avaient donc pas besoin des Français, disaient-ils. Ils avaient une culture et savaient philosopher.

11 Le rapport à l'éducation était donc beaucoup ample et décomplexé. Pour moi, le français n'est pas la langue des Français, c'est ma langue. Un de mes amis dit que c'est un butin de guerre. Mais, pour moi, ce n'est même pas cela. Cela fait seulement partie de la culture que j'ai reçue. Je suis davantage le produit d'un Empire colonial français que d'une Afrique qui serait authentique.

12 Quand je suis arrivé en France pour aller en classe préparatoire, le dépaysement n'a pas été important. Mais ce que j'ai découvert, différent de l'Afrique, c'est que la culture impériale est différente de la culture métropolitaine. Des questions qui n'ont jamais été très importantes pour moi, comme la réflexion sur la race, ont commencé à s'imposer, comme les questions liées à la nature de la culture dans laquelle j'ai grandi. Il s'agit d'une culture très hybride et qui n'est pas présente dans mon éducation.

En arrivant en France, je découvre que je peux me trouver dans une classe dans laquelle je peux compétitionner avec de petits Berrichons ou de petits Alsaciens sur les mêmes thèmes parce que j'ai été élevé de la même manière qu'eux, mais en Afrique. Et l'avantage que j'avais est que je ne parlais pas bien français. Les gens avec qui j'étais en classe disaient tout le temps que je parlais comme un livre. Je connaissais la concordance des temps, je connaissais des mots énormes! Mais je ne pouvais pas avoir une conversation orale normale. J'étais excellent en français parce que j'avais lu plein de choses bizarres.

Comme une langue morte?

14 M. D. : C'est cela.

\section{Comme du latin?}

15 M. D. : Mais, en même temps, c'était drôle parce que c'était un « latin » que les Sénégalais ont toujours parlé. Il y a aussi l'influence de Senghor. C'est un pays où, paradoxalement, il n'y a pas ce que l'on pourrait appeler un créole français ou un pidgin français. Mais il y a un créole ou pidgin portugais qui date du premier Empire portugais.

Quand j'arrive aux États-Unis, cela change complètement. Fondamentalement, dans le système français, on apprend à stocker des connaissances, à les mettre synchrones pour écrire une dissertation. Aux États-Unis, je me suis aperçu que la dissertation est spécifiquement française. Je pense qu'elle n'existe pas ailleurs. La dissertation impose une manière de penser et d'organiser la pensée très différemment que dans un essai ou une thèse, par exemple. La dissertation impose précisément la nécessité de stocker des connaissances. Dans le système américain, on apprend à chercher la connaissance. On apprend où aller pour chercher de la connaissance. Très tôt, dès l'école primaire, quand 
les élèves apprennent la germination des plantes, par exemple, on leur demande de faire germer une plante à partir d'une graine et de décrire comment cela se passe.

En France, quand nous arrivons après le bac à l'université, nous sommes complètement épuisés. Quand l'étudiant américain arrive à l'université, il a une fraîcheur extraordinaire. Tout ce qu'il a appris, il est capable de l'effacer. Il est comme un ordinateur. Il emmagasine.

Nous abordons directement la révolution numérique. Ce que l'Américain apprend, c'est exactement la media literacy actuelle. Maintenant, en un clic entre le professeur et l'étudiant, la connaissance est la même. L'étudiant a même l'avantage de disposer d'une tablette devant lui, à la différence du professeur. L'étudiant peut donc «coincer» le professeur à tout moment. Mais, aujourd'hui, on dit qu'il faut apprendre à aller chercher. C'était donc déjà présent dans le système américain.

18 M. D. : L'étudiant américain n'essaie pas de coincer le professeur. C'est aussi une grande différence.

Il n'y a pas de rivalités de connaissances.

19 M. D. : Oui. Il essaie d'entrer dans une relation dans laquelle il est capable de montrer qu'il a lu ce qu'on lui a dit de lire, qu'il sait de quoi il parle. Avant de venir en classe, mes étudiants me disent ce qu'ils ont lu et le type de questions sur lesquelles nous pouvons discuter. C'est ce que l'élève américain apprend tôt. Ce n'est pas ce que j'ai appris. Je le constate aussi avec mon fils. Je me rappelle lui avoir dit un jour d'apprendre ses tables de multiplication. Il m'a regardé, il m'a dit: "Pourquoi apprendre les tables de multiplication? J'ai une machine.»

20 Mais cela change aussi tout à fait le rapport à la connaissance. Quand Obama a été candidat pour la première fois, mon fils avait douze ans. Il était fan d'Obama. Un jour, il m'a dit : «Je veux être président des États-Unis.» Je l'ai regardé et je lui ai dit: «Tu ne peux pas être président des États-Unis.» « Pourquoi ? ", m'a-t-il demandé. «Tu n'es pas né ici. Et la loi dit que, pour être président des États-Unis... » Il m'a répondu : «Ce n'est pas une raison. On va changer la loi. » Moi, je ne peux pas penser de cette façon. Et c'est ce que l'on apprend aux jeunes Américains, c'est-à-dire de se trouver dans une position où il est capable de pousser la conversation.

21 Le numérique met aujourd'hui à leur disposition des connaissances. Ils peuvent toujours vérifier ce qu'on leur dit. Et, après vérification, ils peuvent se faire une idée du contenu et de la nature de la discussion dans laquelle ils sont engagés. L'idée de débat et de débattre est beaucoup plus importante. Encore une fois, je le constate avec mon fils. Quand il vient en France je suis obligé de lui expliquer que, bien qu'il soit au lycée, il ne peut pas engager une conversation en exprimant son désaccord à son professeur.

Le système français forme et, au sens strict, "conforme» ?

22 M. D. : Oui. Aux États-Unis, il y a d'autres habitudes. L'élève pose des questions qui illustrent visiblement le doute qu'il a sur ce qu'on lui a dit.

Vous avez une vision originale, puisque vous avez connu de l'intérieur une diversité de systèmes éducatifs: américain, français, africain. Pour vous, quelles sont les conséquences de la mondialisation sur l'éducation?

23 M. D. : En fait, le secteur le moins touché par la globalisation est l'éducation et ce, pour une raison très simple. La plupart des sociétés considèrent que le citoyen se forme dans le secteur éducatif - c'est la thèse de Jules Ferry. C'est là que la nation s'apprend. C'est là que le récit de la nation est appris et inculqué. La langue devient le vecteur le plus important. 
Aujourd'hui, c'est la situation dans laquelle se trouvent les Français. La France se trouve dans une situation paradoxale. Pour enseigner à Sciences Po, j'enseigne en anglais. L'anglais est la langue de la globalisation. Mais ce que l'on perd fondamentalement dans ce rapport est que, contrairement à ce que l'on croit, l'anglais qui est utilisé par toi, par moi, cet anglais académique n'est pas l'anglais des sociétés anglophones. Il existe plusieurs anglais. L'anglais de la littérature indienne est différent de celui de la littérature kényane. En fait, les migrants jouent un rôle essentiel à ce titre. Certaines régions islamiques ont fondamentalement changé. Par exemple, à partir du moment où les Soudanais ont commencé à migrer en Arabie Saoudite, ils sont passés d'un islam très indigène et très local à un islam beaucoup plus global, avec des exigences beaucoup plus autoritaires.

On retrouve là ce qui se retrouve en Occident: la capacité des gens à reformater les cultures. Il s'agit non seulement de les reformater mais de participer à leur propre production. En quoi New York est une ville américaine? C'est une question qu'on pourrait se poser. Il y a une vingtaine d'années, un groupe de rap sénégalais, Positive Black Soul disait dans une de ses chansons, assez extraordinaire : « New York et Paris sont des banlieues de Dakar. » Mais ce n'est pas traduit en termes d'apprentissages et de connaissances. C'est le problème. Le problème est que nous vivons complètement immergés dans un monde qui a une autre langue, une autre démarche, d'autres façons de faire les choses. Mais l'école reste, comme on dit en France, laïque et républicaine.

Selon vous, y a-t-il aujourd'hui des enjeux éducatifs communs à tous les systèmes éducatifs du monde? Vous dites que le système éducatif est ce qui est le moins mondialisé et le plus rattaché à la nation. Mais, aujourd'hui, du fait de la globalisation, y aurait-il des enjeux internationaux au niveau éducatif?

M. D. : La frontière entre l'instruction et l'éducation s'est élargie. On est techniquement globalisés. C'est pour cette raison que la plupart des gens qui dirigent les mouvements dits radicaux islamiques sont des ingénieurs. Ce sont des gens qui sont au fait de la technologie et complètement ancrés dans une tradition que contredit cette science qu'ils détiennent. Précisément, l'instruction ne porte pas le projet éducatif. Par exemple, surtout dans le monde musulman, ceux que l'on appelle les musulmans radicaux ont compris plus rapidement que d'autres les enjeux éducatifs de la globalisation. Avant que ces groupes ne deviennent des groupes armés, la première étape de leur action a toujours été d'abord une intervention éducative. Eux ne confondaient pas l'instruction et l'éducation. Ils donnaient une instruction religieuse qui fixait une direction, un objectif, une façon de vivre sa vie et le monde. Ils donnaient cette instruction qui permet effectivement de trouver un travail, mais aussi de fabriquer des bombes, de créer des sites web pour y mettre des informations. C'est leur grande réussite.

C'est ce qui se retrouve chez les intégristes, les pentecôtistes ou les évangélistes. Le très grand succès des mouvements religieux chrétiens en Afrique et en Amérique Latine est lié au fait qu'ils ont un projet éducatif porté par une extraordinaire maîtrise de la technologie du temps du monde.

La seule solution pour y arriver aujourd'hui est probablement d'accepter la créolisation, c'est-à-dire d'accepter l'idée qu'il n'y a pas qu'une seule culture capable de fournir une orientation au monde. C'est pour moi la chose la plus essentielle. On ne pourra y arriver du point de vue éducatif qu'en respectant une chose - ce qui n'est pas le cas aujourd'hui. Le projet éducatif ne peut pas porter une sorte d'œcuménisme culturel. Nous refusons aujourd'hui de reconnaitre que nous vivons dans un monde pluriel. Or le pluralisme est 
aujourd'hui ce qui doit permettre de repenser un monde pluriel. C'est pourquoi ce temps du monde, aujourd'hui en germination, ne peut être que le temps du monde de l'échange.

Senghor avait raison sur ce point. Et c'est un moyen de revenir à l'Inde. Le grand historien indien contemporain, Narajit Guha, a été à l'origine, après la révolution des Annales en France, de la plus grande révolution historiographique des subaltern studies. Il était marxiste maoïste. Il dit aujourd'hui que Tagore avait raison et que la seule manière de retourner au pluralisme est de refuser une histoire définie seulement par la politique. C'est vrai que l'histoire occidentale de l'État nation - est d'abord une histoire politique. Guha dit qu'il faut écrire une histoire de la vie quotidienne. C'est ce qui permet de reconnaître le pluralisme.

L'école de la nation est passée au-dessus de cultures locales, que ce soit au Sénégal ou partout ailleurs. L'africanisation des programmes a aussi fait apprendre une certaine Afrique. Ce n'est pas I'Afrique dans sa pluralité. La sortie de ce temps-là n'est-elle pas l'opportunité d'éduquer à un monde créolisé et pluriel ? De quelle manière cela peut-il se faire?

M. D. : C'est le grand problème des Africains. Ils créent un récit national, mais sans pouvoir soumettre les récits locaux. Ils créent un récit national parce qu'ils considèrent que les récits locaux sont nécessairement antagonistes, ce qui est faux. C'est là que réside le problème de l'Afrique.

\section{Sont-ils antagonistes entre eux ou avec le discours national ?}

M. D. : Le discours national crée des antagonismes parce que c'est un discours qui n'est même pas fédérateur. C'est un discours qui se produit au-dessus des autres discours en assumant que le rapport entretenu par ces différentes cultures ne peut être qu'antagonique. C'est faux. Nous assistons à la production d'un récit national qui est constamment déconstruit par des récits locaux. Cela veut dire que ces États n'existent pas et ne sont pas des nations. Or c'était possible. En Afrique, on a décidé qu'il était impossible de créer une culture nationale en reconnaissant la diversité. C'est ce qui est important. Comment créer un espace dans lequel peut avoir lieu un dialogue constant?

Pensez-vous que cela peut commencer par l'école ou l'université ?

31 M. D. : Bien sûr. Nous disposons aujourd'hui de l'expertise pour le faire. Je parlais de mes enfants aux États-Unis. Ils viennent d'une école francophone. Ils peuvent entrer très rapidement dans un système anglophone tout en ayant la capacité de retourner dans le monde d'où ils viennent. Nous ne nous rendons pas compte que nous avons des identifications stratégiques. C'est pour cette raison que je n'emploie pas le terme d'«identité » qui est figé. Dans une certaine mesure, les États-Unis sont arrivés à faire cette sorte de basculement. On dit aux étudiants américains qu'il faut apprendre d'autres cultures ethniques. Les Américains disposent du concept de «fusion». C'est ce qui montre que c'est effectivement possible.

Des Sénégalais qui font du thiebou dieune, du riz au poisson, sont aujourd'hui capables de faire du fast-food de riz au poisson. C'est la créolisation. Quand j'étais au Sénégal, je n'aurais jamais imaginé qu'il était possible de prendre son téléphone pour commander un htiebou dieune. Cela se fait aux États-Unis parce que, précisément, les gens utilisent leur identification de manière très stratégique.

Quand je rédige mon rapport annuel, cela ne concerne pas seulement mon enseignement, mes recherches et mes publications, mais aussi ce qu'ils appellent « community service ». Je suis sénégalais. Il y a une communauté sénégalaise. J'en fais partie. Je suis historien. Je 
fais partie de la communauté des historiens. Je suis membre de l'Association des historiens. J'enseigne les african studies. Je fais partie de cette communauté des african studies. Je suis africain. Je peux dépasser les limites des Sénégalais. Je suis noir. J'intègre les Africains américains dans un territoire beaucoup plus large. Ce territoire comprend Sénégalais, Africains, Africains américains. Il s'agit d'un territoire unique (les Noirs) et aussi des territoires de différenciation. Je suis musulman aussi. Mes copains américains pensent que je suis un Black French parce qu'ils ne peuvent pas imaginer que je vienne d'Afrique. Pour expliquer qui je suis, ils font ce détour par la France par rapport à la façon dont je m'habille et d'autres choses. C'est vrai, cela constitue une communauté de gens qui font certaines choses qui sont reconnues légalement dans les universités publiques.

Quand j'enseignais au Michigan, je recevais des centaines de mails d'élèves de lycées ou de collèges. Ils me disaient : « On nous a dit ça de l'Afrique. On ne le croit pas. » Quand j'ai été recruté par l'université du Michigan, tout le monde savait que j'étais là, ce que je faisais et que j'étais au service des gens qui avaient des questions à poser.

L'école peut le faire, mais en faisant d'abord une espèce de révolution idéologique. Je ne suis pas nécessairement opposé au récit national, mais je suis opposé à un récit national univoque et unilinéaire. Je suis favorable à un récit national qui peut engager les autres, capable de s'adapter aux défis que lui posent d'autres récits à l'intérieur et aux défis que lui posent les récits des autres nations. Pour un historien, c'est un point très intéressant. Avec les empires, c'est tout à fait extraordinaire. Les empires administrent le pluralisme linguistique, culturel, etc. L'Empire ottoman a pu administrer des orthodoxes chrétiens, des chrétiens romains, des musulmans de toutes les obédiences, des Arméniens, des Juifs. L'une de mes collègues, Karen Barkey, appelle cela l'« administration du pluralisme ». Elle a publié un livre sur l'Empire ottoman (Empire of Difference). Il y a aussi un livre de Fred Cooper publié en français (Empires). Cela s'appelle la politique de la différence.

Il existe un débat dans le monde anglo-saxon entre ceux qui parlent de politics of difference et ceux qui parlent de rules of difference ce qui correspond à la façon dont l'empire colonial, surtout britannique, impose le maintien des frontières linguistiques et culturelles. Dans ce cas, les gens sont gouvernés sur la base de ce qui est défini comme leur culture propre. Il s'agit d'un type d'État qui repose sur les fragments. C'est pour cette raison que ce type de structures politiques reconnaît l'hégémonie politique et la domination politique sans s'intéresser à l'assimilation ou à la transformation des sociétés dominées dans la société principale. Aujourd'hui, nous avons une vision de la nation qui est celle de l'homogénéisation.

Y a-t-il une incapacité française particulière à intégrer la différence ?

M. D. : En fait, il y a un mythe autour de cette question, mais je ne pense pas que ce soit vrai. Un vieil écrivain ivoirien, Bernard Dadié, a écrit deux livres extraordinaires à propos de ces questions (Climbié et Un Nègre à Paris). Il décrit la logique qui fait que le discours français est contredit par la pratique coloniale. Il dit: «Ils nous disent qu'on est ignorants, mais ils ne veulent pas nous ouvrir leurs bibliothèques. Ils ne veulent pas que nous lisions. Ils disent qu'on est des sauvages par la manière dont on s'habille, mais quand on s'habille à l'européenne, ils disent qu'on ressemble à des singes savants. » En fait, cette logique d'une offre théorique de la mission civilisatrice, mais d'une pratique qui dévie complètement cette offre, est probablement l'une des plus grandes forces de l'idéologie française. Elle est très forte. Elle fait la même chose avec la race et le racisme en disant ne pas les connaître. La fonction principale de cet écart établi entre le discours 
et la pratique est de garder intacte la fiction d'une société dans laquelle la race n'a pas d'importance.

Quelle est la racine de cela?

38 Américains avaient fait une révolution parfaite, avec la liberté et l'égalité, mais qu'il ne s'agissait pas d'une révolution universelle, mais d'une révolution pour les Américains, alors que la révolution américaine met en avant que tous les hommes sont créés égaux. C'est inscrit dans la Déclaration d'Indépendance et pas dans la Constitution. Dès 1776, l'universel est posé.

L'idéologie française accepte que d'autres nations peuvent avoir plein de qualités, mais ils revendiquent le privilège de l'universalité. Étrange paradoxe. C'est ce que l'on retrouve dans I'Histoire de France de Lavisse.

44

Tréma, 40 | 2013 
Et, quand on fait quelque chose de bien, on doit être français.

M. D. : Bien sûr.

Lors d'une émission télévisée, l'auteur Jean-Paul Brighelli à qui on faisait reconnaître qu'il pouvait y avoir quelque chose d'universel dans le texte du Coran, répondait: "C'est que Mohamed devait être, en quelque sorte, français. »

46

M. D. : Bien sûr. C'est l'idée qu'il existe un modèle unique d'universalité, de laïcité... C'est vrai que les Français l'ont toujours bien « vendu ».

Pour conclure, s'il fallait qualifier l'école idéale de demain?

M. D. : Une école du pluralisme, pour de véritables citoyens - des citoyens du monde. Il ne s'agit pas d'effacer la citoyenneté nationale, mais d'y ajouter une citoyenneté locale et, à celle-ci, d'ajouter d'autres connexions, d'autres lectures, d'autres récits, d'autres histoires.

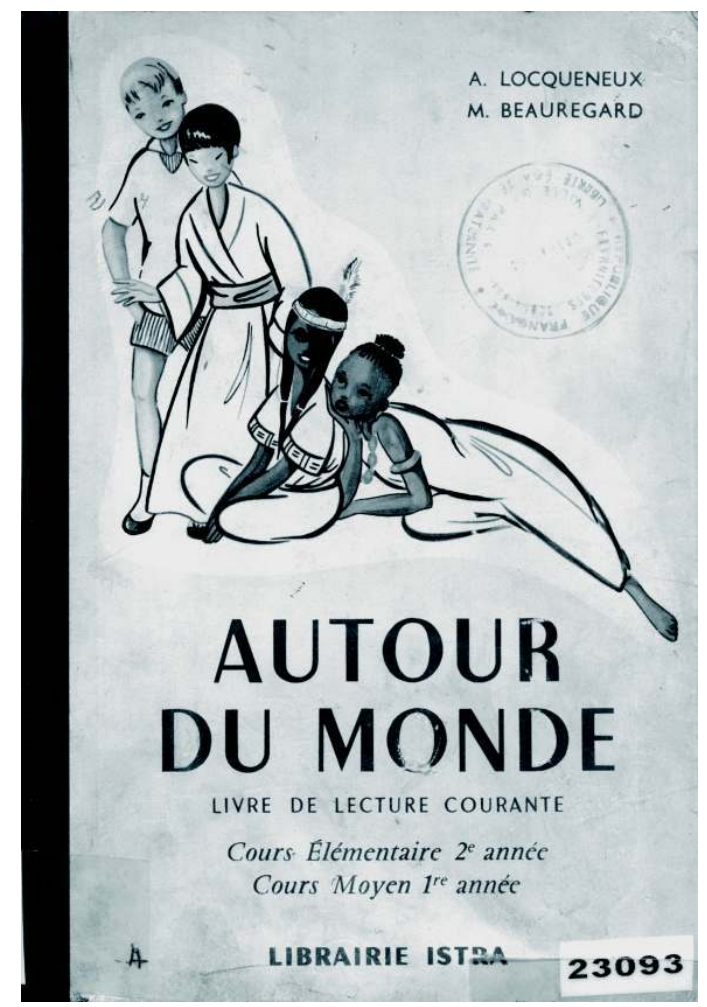

Autour du monde. Locqueneux, A. ; Beauregard, M., Paris : Librairie Istra. 1962. Source Cedrhe 23093. Couverture.

\section{AUTEUR}

\section{MAMADOU DIOUF}

Professeur d'histoire et directeur de l'Institut d'études africaines à la Columbia University à New York 